Pesq. Vet. Bras. 37(2):121-128, fevereiro 2017 DOI: 10.1590/S0100-736X2017000200005

\title{
Avaliação da eficácia da lasalocida e de alguns fatores epidemiológicos de Eimeria spp. parasitando bezerros Nelore mantidos em regime de pastejo ${ }^{1}$
}

\author{
Leonardo Bueno Cruvinel ${ }^{2}$, Dyego Gonçalves Lino Borges ${ }^{3}$, João Eduardo \\ Nicaretta ${ }^{2}$, Thiago Souza Azeredo Bastos ${ }^{2}$, Elio Moro ${ }^{4}$, Rogério Dantas Gama ${ }^{5}$, \\ Fernando de Almeida Borges ${ }^{3}$ e Welber Daniel Zanetti Lopes ${ }^{5 *}$
}

\begin{abstract}
Cruvinel L.B., Borges D.G.L., Nicaretta J.E., Bastos T.S.A., Moro E., Gama R.D., Borges F.A. \& Lopes W.D.Z. 2017. [Effectiveness of lasalocid assessment and some epidemiological factors of Eimeria spp. parasitizing Nellore calves kept on pasture.] Avaliação da eficácia da lasalocida e de alguns fatores epidemiológicos de Eimeria spp. parasitando bezerros Nelore mantidos em regime de pastejo. Pesquisa Veterinária Brasileira 37(2):121-128. Instituto de Patologia Tropical e Saúde Pública, Universidade Federal de Goiás, Goiânia, G0 74605-050, Brazil. E-mail: wdzlopes@hotmail.com

The main importance of eimeriosis in cattle is due to lower performance shown with the disease in its sub-clinical form. This study evaluated the efficacy of lasalocid used against Eimeria spp. parasitizing calves. We also evaluated the weight gain of calves submitted to different treatments and analyzed some epidemiological factors that might interfere with Eimeria infection; 288 calves were used in the study. The calves of treatment 1 received protein mineral salt in low consumption without lasalocid, while the calves of treatment 2 received protein mineral salt on low consumption with lasalocid, administered orally to 4 to 10-month-old calves. Harvest of feces and weight control was made on days 0 (before the start of the experiment), at weaning, and 30 and 60 days after weaning (DAW). Evaluation of some epidemiological factors which could be related to infection by Eimeria spp. of the calves, such as weaning, sex and time of year, were analyzed, taking into account the results regarding the 144 calves of the control group. Nine species of Eimeria were identified in descending order: E. brasiliensis, E. wyomingensis, E. bovis, E. canadian, E. zuernii, E. auburnensis, E. ellipsoidalis, E. pellita and E. cylindrica. Unexpectedly, decrease in parasite load could be observed after weaning. Even the farm did not adopt management measures aimed for greater productivity, as Artificial Insemination in Fixed Time, which in turn ends up with increase of the number of births and animal unit per hectare at a certain period of year, high parasitism of coccidia was diagnosed in calves of the control group. Oocyst counts per gram (OPG) of calves treated with lasalocid were significantly lower $(\mathrm{P} \leq 0.05)$ in the control group. The compound achieved $\geq 95 \%$ efficacy against the parasite in question. At the end of the study, calves fed lasalocid gained on average $7.2 \mathrm{~kg}(\mathrm{p} \leq 0.05)$ more than calves in the control group. For a farm that aims to sell calves soon after weaning, is recommended to start treatment with lasalocid, with the creep-feeding, from an age of three months on, since the weight gain calves treated with lasalocid was significantly $(\mathrm{p}=0.05)$ higher compared with the weight gain of the control group after five months of treatment. The difference in weight
\end{abstract}

\footnotetext{
${ }^{1}$ Recebido em 15 de abril de 2016.

Aceito para publicação em 30 de setembro de 2016.

${ }^{2}$ Programa de Pós-Graduação em Ciência Animal, Universidade Federal de Goiás (UFG), Av. Esperança s/n, Campus Samanbaia, Goiânia, GO 74690-900, Brasil.

${ }^{3}$ Departamento de Medicina Veterinária, Universidade Federal de Mato Grosso do Sul (UFMS), Av. Filinto Müller 2443, Ipiranga, Cx. Postal 549,
}

\footnotetext{
Campo Grande, MS 79074-460, Brasil.

${ }^{4}$ Zoetis Saúde Animal, Rua Henri Dunant 1383, Morumbi Corporate Ed. Diamond Tower, 4o andar, São Paulo, SP 04709-110, Brasil.

${ }^{5}$ Departamento de Parasitologia, Instituto de Patologia Tropical e Saúde Pública, Universidade Federal de Goiás (IPTSP/UFG), Rua 235 s/n, Setor Leste Universitário, Goiânia, GO 74605-050, Brasil. *Autor para correspondência: wdzlopes@hotmail.com
} 
gain of calves treated with lasalocid compared with caves in the control group may also be partially related to the infection by Eimeria spp., as discussed in this paper.

INDEX TERMS: Lasalocid, epidemiology, Eimeria spp., calves, weaning, eimeriosis, effectiveness.

RESUMO.- A principal importância da eimeriose em bovinos, se deve ao baixo desempenho produtivo que os animais demonstram quando esta enfermidade apresenta-se sob a forma sub-clínica. Como objetivos, o presente trabalho avaliou a eficácia do uso da lasalocida sódica contra espécies de Eimeria spp. parasitando bezerros; avaliou também o desempenho ponderal dos animais submetidos aos diferentes tratamentos e analisou alguns fatores epidemiológicos que possam interferir na infecção por Eimeria nos bezerros. Foram utilizados 288 bezerros no dia 0 do estudo. Os animais pertencentes ao tratamento 01 receberam sal mineral proteinado de baixo consumo sem adição de lasalocida, enquanto que os bezerros do Tratamento 02 sal mineral proteinado de baixo consumo, com adição de lasalocida sódica, administrado via oral para bezerros dos quatro/cinco/seis meses até dez meses de idade. Colheita de fezes e pesagem dos animais foram realizadas nos dias 0 (antes do início do experimento), na desmama, 30 e 60 dias após desmama (DPD). A avaliação de alguns fatores epidemiológicos que pudessem ser relacionados com a infecção por Eimeria spp nos bezerros, como o desmame, sexo e época do ano, foram analisados neste estudo, levando-se em consideração os resultados encontrados durante todo estudo, para os 144 animais pertencentes ao grupo controle. Foram identificadas nove espécies de Eimeria nos bezerros em ordem decrescente: E. brasiliensis, E. wyomingensis, E. bovis, E. canadenses, E. zuernii, E. auburnensis, E. ellipsoidalis, E. pellita e E. cylindrica. Inesperadamente, diminuição na carga parasitária dos animais pode ser observada após o desmame. Mesmo a fazenda não adotando medidas de manejo que visam maior produtividade como a Inseminação Artificial em Tempo Fixo, que por sua vez acaba aumentando o número de nascimentos e unidade animal/hectare em uma determinada época do ano, elevado parasitismo pelo coccídio em questão foi diagnosticado nos bezerros pertencentes ao grupo controle. Talvez a época do ano em que o estudo foi realizado pode ter influenciado neste aspecto. As contagens de oocistos por grama (OoPG) de fezes para Eimeria dos animais tratados com lasalocida foram estatisticamente inferiores $(\mathrm{P} \leq 0,05)$ as do grupo controle após o início do estudo. 0 composto alcançou eficácia $\geq 95 \%$ contra o parasito em questão. No final do estudo, os animais que receberam lasalocida ganharam em média, 7,2kg a mais $(\mathrm{P} \leq 0,05)$ que os bezerros pertencentes ao grupo controle. Em propriedades que tem como objetivo a venda de bezerros logo após a desmama, recomenda-se o início do tratamento com a lasalocida, junto ao creep-feeding, a partir de três messes de idade, uma vez que diferencial no ganho em peso médio dos bezerros tratados foi significativamente $(\mathrm{P} \leq 0,05)$ mais elevado, em comparação ao grupo controle, após cinco meses de tratamento com o referido composto. Apesar de a lasalocida ser utilizada como um aditivo alimentar para animais, a diferença no ganho em peso vivo médio entre animais tratados com a lasalocida, em comparação a animais pertencentes ao grupo contro- le, também pode ser relacionada, em partes, a infecção dos animais por Eimeria spp., conforme discutido neste artigo, entretanto, futuros estudos devem ser conduzidos para comprovar esta hipótese.

TERMOS DE INDEXAÇÃO: Lasalocida, epidemiologia, Eimeria spp., bezerros, desmame, eimeriose, eficácia.

\section{INTRODUÇÃO}

Coccidiose é nome que se da para as enfermidades parasitárias ocasionadas por protozoários pertencentes à Sub-classe COCCIDIA, sendo representados principalmente em ruminantes por: Cryptosporidium spp., Sarcocystis spp., Toxoplasma gondii e Eimeria spp. No entanto, este termo é mais comumente utilizado para se referir as parasitoses ocasionadas por Eimeria, que por sua vez pode acometer aves, bovinos, ovinos, caprinos, suínos, eqüinos e coelhos. Dentre estas espécies animais, os bovinos, juntamente com os ovinos e caprinos, são mais comumente infectados pelo protozoário em questão, que por sua vez podem desencadear efeitos deletérios aos animais portadores e conseqüentemente prejuízos aos produtores (Lopes et al. 2014). De acordo com Aumont et al. (1984) e Lima (2004), a principal importância da eimeriose em ruminantes, se deve ao baixo desempenho produtivo que os animais demonstram quando esta enfermidade se apresenta sob a forma sub-clínica. Segundo os mesmos autores, estes animais necessitam de um tempo adicional para atingir o peso equivalente aos animais não infectados, e a mortalidade de animais por Eimeria spp., apesar de rara, pode acontecer em jovens com grau de parasitismo elevado. Cavalcante et al. (2009) relatam ainda que, a queda na produtividade pode representar maior prejuízo econômico para o produtor do que a própria mortalidade, que raramente ultrapassa $10 \%$ de um rebanho. Os pesquisadores Lima (2004) e Nogueira et al. (2009) reforçam a necessidade em se realizar mais estudos que visem conhecer os aspectos epidemiológicos desta enfermidade, com a identificação das espécies envolvidas, entender os possíveis danos que Eimeria spp. pode ou não ocasionar em determinada categoria animal, e, para que na sequencia, alguma estratégia de prática de manejo seja pesquisada e aplicada, quando necessário, desde que esta ferramenta seja efetiva, e economicamente viável para os produtores de bovinos.

Dentre os ativos utilizados em bovinos contra Eimeria no Brasil, destacam-se as sulfonamidas (Lopes et al. 2014), o amprólio (Nogueira et al. 2010), os antibióticos ionóforos como a monensina, salinomicina e lasalocida (Lima et al. 2009), e o toltrazuril (Philippe et al. 2014). A atuação dos ionóforos no controle de Eimeria spp ocorre pela perturbação do fluxo normal de íons na membrana celular, conduzindo a alterações na pressão osmótica e eletrolítica no interior da célula, causando a morte do parasito nos estágios de esporozoíto e merozoíto. Dentre os ionóforos, a lasalocida é um metabólito microbiano produzido por es- 
pécies de Streptomyces spp. (Nogueira et al. 2009). Pode ser utilizada tanto para o controle da eimeriose, quanto como aditivo em alimentos para animais, com o propósito de aumentar a eficiência alimentar e, consequentemente, otimizar o desenvolvimento e o ganho em peso dos animais, devido às alterações na fermentação ruminal (Rutkowski \& Brzezinski 2013).

Apesar do uso terapêutico de antibióticos ionóforos ter se difundido de forma expressiva nos últimos anos, ainda são escassos os trabalhos, conhecidos por nós, que objetivaram avaliar alguns esquemas/manejos de tratamentos que sejam eficientes no controle de Eimeria em determinadas categorias, e que consequentemente demonstrem relação custo beneficio positiva em sua utilização. É notório que após o aumento na utilização destes ativos via ração, surgiu, consequentemente, o aumento no risco de intoxicação dos animais. Tal fato ressalta ainda mais, a importância em se realizar pesquisas para se determinar a dosagem específica para cada espécie e categoria animal (Nogueira et al. 2009).

Por estes motivos supracitados, o presente trabalho teve como objetivos: avaliar a eficácia do uso da lasalocida sódica (administrada na dose de $1 \mathrm{mg} / \mathrm{kg}$, via sal mineral proteinado junto ao creep-feeding), contra espécies de Eimeria spp. parasitando bezerros Nelore, naturalmente infectados e mantidos em regime de pasto; identificar as espécies de Eimeria; avaliar o desempenho ponderal dos animais submetidos aos diferentes tratamentos e analisar alguns fatores epidemiológicos que possam interferir na infecção por Eimeria nos bezerros.

\section{MATERIAL E MÉTODOS}

Local e animais. 0 presente estudo foi realizado entre maio e novembro de 2014, na Fazenda Entre Rios, localizada no município de Aruanã, estado de Goiás, Centro Oeste do Brasil. A região é caracterizada por clima tropical, quente e semiúmido, com verão chuvoso e períodos secos durante o inverno. Este trabalho foi submetido ao comitê de ética da Universidade Federal de Goiás, tendo seu parecer aprovado sob processo de número 0023/14.
Foram utilizados 288 bezerros, lactentes, da raça Nelore, machos e fêmeas, com idade entre quatro e seis meses (peso vivo entre $140 \mathrm{Kg}$ e $180 \mathrm{Kg}$ ) no dia 0 do estudo, e naturalmente infectados por Eimeria spp. Todos os animais (vacas e bezerros) foram previamente vacinados contra importantes enfermidades (Febre Aftosa e Clostridioses), de acordo com o manejo sanitário estabelecido previamente na fazenda. Foram incluídos no estudo somente bezerros em bom estado nutricional, sem qualquer tipo de doença no dia da randomização (Dia 0) e que não haviam sido suplementados ou recebido ionóforos ou qualquer outro composto químico com ação contra Eimeria desde o nascimento. Os animais foram desmamados com oito meses de idade, e o estudo foi encerrado para cada bezerro, quando estes atingissem 10 meses de idade (60 dias pós-desmama).

Grupos e tratamentos. Para o estudo, foram utilizados seis piquetes homogêneos de aproximadamente 25 hectares/cada, contendo gramínea tropical composta predominantemente por Brachiaria MG5 e Pietã. Os bezerros foram distribuídos em dois grupos de tratamentos (T01 e T02), conforme descrito no próximo parágrafo, com três repetições de área/piquete cada, homogêneos entre si. A constituição dos dois grupos de tratamentos (T01 e T02) foi do tipo casualizada e obedeceu ao seguinte critério: na repetição de área número 1 (piquetes 1 e 2), foram alocadas, em cada piquete, 48 vacas e suas respectivas crias (24 machos e 24 fêmeas); os dois pastos pertencentes à repetição de área número dois da mesma forma, e assim sucessivamente para completar a repetição de área número três (Fig.1). Os animais foram alocados de tal forma nos piquetes, que em cada repetição de área continha números equivalentes de bezerros machos e fêmeas, com idades de quatro, cinco e seis meses. A determinação em qual piquete ficaria cada tratamento (T01 ou T02), dentro de uma repetição de área, foi determinada por meio de sorteio.

Após a randomização, os animais permaneceram nos seus respectivos piquetes durante todo o experimento, com acesso a água natural e cochos de sal mineral à disposição para vacas e bezerros. A partir do dia zero, (após a randomização), os bezerros de ambos os grupos receberam suplementação de sal proteinado de baixo consumo por meio do sistema creep-feeding. Os animais pertencentes ao tratamento 01 , receberam sal mineral proteinado de baixo consumo (150 gramas/cabeça/dia) sem adição de lasalocida, enquanto que os bezerros do T02 receberam sal mi-
Repetição de Área 2

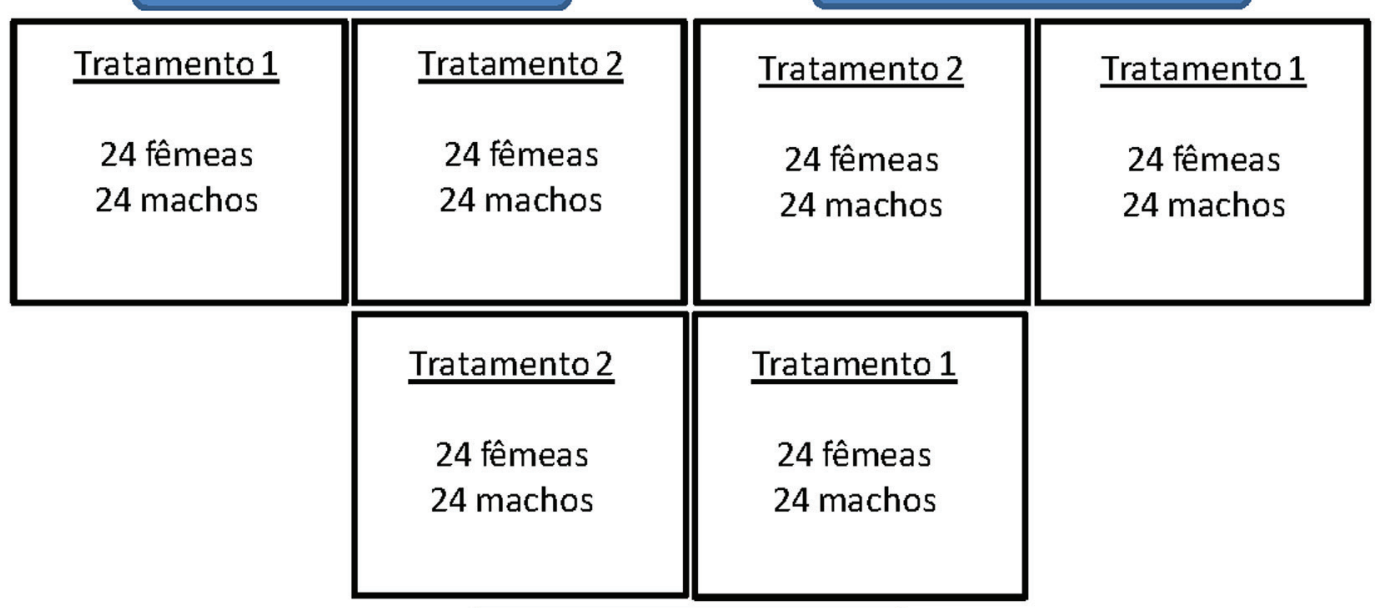

Repetição de Área 3

Fig.1. Delineamento experimental ilustrando a alocação dos grupos de tratamento (T01 e T02) nas diferentes repetições de áreas. 
neral proteinado de baixo consumo (150 gramas/cabeça/dia), com adição de lasalocida sódica (Taurotec ${ }^{\circledR} 15 \%$ - Zoetis Brasil), administrado via oral (adicionado no concentrado), na dose de 1 $\mathrm{mg}$ de principio ativo por $\mathrm{kg}$ de peso vivo, equivalendo a 1,3g de lasalocida para cada $200 \mathrm{~kg}$ de peso vivo dos quatro/cinco/seis meses até dez meses de idade. Além disso, diariamente todos os animais foram observados por um veterinário, com o objetivo de constatar possíveis diarréias que pudessem ser atribuídas à infecção por Eimeria nos bezerros.

É importante frisar que, logo após o desmame (que aconteceu quando os animais atingiram oito meses de idade), estes bezerros permaneceram no mesmo piquete de origem até completarem os 10 meses de idade (dois meses após a desmama), quando na sequencia, estes animais foram excluídos do estudo.

Colheita de fezes e cálculo da eficácia. Amostras de fezes foram colhidas diretamente da ampola retal dos animais nos dias 0 (antes do início do experimento), na desmama, 30 e 60 dias após desmama (DPD). Desta forma, levando em consideração que no dia 0 do estudo havia animais de quatro a seis meses de idade, que os bezerros foram desmamados com oito meses, e que o estudo foi encerrado quando estes bezerros completassem 10 meses de idade, houve colheita de fezes $60,90,120,150$ e 180 dias pós-início do estudo (DPIE). Em outras palavras, as colheitas de fezes realizadas no $60^{\circ}$ DPIE foram provenientes dos bezerros que tinham seis meses de idade no dia 0 do estudo, enquanto que as colheitas realizadas no $180^{\circ}$ DPIE eram pertencentes aos animais que apresentavam quatro meses de idade no dia 0 . As fezes foram analisadas quanto a presença de oocistos por grama (OoPG) de fezes segundo a técnica descrita por Gordon \& Whitlock (1939), modificada.

0 percentual de eficácia obtido pela lasalocida, em cada data de observação, foi calculado de acordo a fórmula descrita a seguir:

Média de OoPG do grupo controle no dia X Percentual $=\frac{\text { - Média de OoPG do grupo tratado no dia } \mathrm{X}}{\text { Média de OoPG do grupo controle no dia X }} \times 100$ de eficácia Média de OoPG do grupo controle no dia X

Identificação das espécies de Eimeria. Um pool das amostras de fezes obtidas no dia 0, na desmama, 30 e 60 dias pós-desmama, dos animais controle e tratados, foram utilizadas para identificar quais as espécies de Eimeria que estavam envolvidas no estudo. As amostras de fezes diagnosticadas como positivas pela técnica descrita por Gordon \& Whitlock (1939), foram processadas utilizando-se o método de centrifugo-flutuação em solução de açúcar (Ueno \& Gonçalves 1998). Na seqüência, estas amostras foram filtradas utilizando-se uma peneira com gaze dobrada e o filtrado posteriormente centrifugado a $250 \mathrm{~g}$ por 10 minutos. Em seguida, este filtrado obtido após a centrifugação foi colocado em uma placa de petri contendo solução de dicromato de potássio 2,5\% $\left(\mathrm{K}_{2} \mathrm{Cr}_{2} \mathrm{O}_{7}\right)$ para estimular a esporulação dos oocistos em laboratório. Depois de dez dias, os oocistos foram recuperados por centrifugação em solução saturada de açúcar $(250 \mathrm{~g}$ por cinco minutos), seguida de uma lavagem em água destilada. Os oocistos esporulados foram concentrados realizando-se outra centrifugação de $250 \mathrm{~g}$ por 10 minutos, e posteriormente foram estocados a $4^{\circ} \mathrm{C}$ em uma solução de dicromato de potássio. Cem oocistos encontrados foram mensurados para identificação da espécie de Eimeria, utilizando um microscópio de luz com sistema computadorizado LAS Leica. Procedeu-se à identificação das espécies de acordo com a forma, cor, presença ou ausência de micrópila e capuz micropilar, e pelo tamanho dos oocistos, além das características morfológicas dos esporocistos, seguindo critérios estabelecidos por Duszynski \& Wilber (1997) e Daugschies \& Najdrowski (2005).

Pesagem dos animais e avaliação dos aspectos epidemiológicos de Eimeria. Com o objetivo de avaliar o desenvolvimen- to ponderal dos bezerros, pesagens foram realizadas no dia 0 , na desmama e 60 dias pós-desmama. 0 ganho em peso vivo (GPV) dos animais foi calculado com base no peso inicial dos bezerros realizado no dia 0 .

A avaliação de alguns fatores epidemiológicos que pudessem ser relacionados com a infecção por Eimeria spp. nos bezerros, como o desmame, sexo e época do ano, foram analisados neste estudo, levando-se em consideração os resultados encontrados durante todo estudo, para os 144 animais pertencentes ao grupo controle (que não receberam nenhuma medição contra Eimeria spp.).

Para anular o efeito de uma possível interferência de nematódeos gastrintestinais no presente estudo, todos os bezerros foram tratados com Moxidectina 200 $\mu \mathrm{g} / \mathrm{kg}$ (Cydectin - Zoetis Brasil) no inicio do experimento (dia 0 ) e depois a cada 45 dias até o término do estudo. Contagens de ovos por grama de fezes foram realizadas (Gordon \& Whitlock 1939), nas mesmas datas experimentais citadas anteriormente para quantificação dos helmintos gastrintestinais (estrongilídeos).

Análise estatística. Em relação à análise dos dados, inicialmente para verificar se a distribuição dos animais por peso vivo e OoPG foi homogênea entre os tratamentos, foram realizadas análises por meio do procedimento ANOVA do programa SAS (1989-1996) (SAS Institute Inc., Cary, NC, USA). As variáveis dependentes (peso vivo e contagem de OoPG) foram analisadas por meio do procedimento MIXED do programa SAS. O modelo incluiu os efeitos fixos de sexo, tratamento, tempo de colheita e suas interações. Para cada variável, os dados foram testados quanto à normalidade e a homogeneidade das variâncias. Os modelos estatísticos incluíram os efeitos de bloco (piquete), tratamento e interação tratamento e piquete.

Diferenças entre os tratamentos foram avaliadas ao nível de significância de $5 \%(P \leq 0,05)$.

\section{RESULTADOS}

Nenhum sinal de anormalidade ou evento adverso foi observado nos animais antes e após a administração da lasalocida. Além disso, os bezerros, ao longo do período em que o estudo foi realizado, não demonstraram sinais clínicos de eimeriose, apesar de inúmeros animais pertencentes ao grupo controle terem apresentado uma carga parasitária entre 3.000 e 10.000 OPG. Por este motivo, a forma encontrada da doença diagnosticada foi considerada como eimeriose sub-clínica.

Durante o estudo, foram identificados oocistos de nove espécies de Eimeria nos bovinos: E. zuernii, E. ellipsoidalis, E. cylindrica, E. bovis, E. canadenses, E. wyomingensis, E. auburnensis, E. brasiliensis, E. pellita. É importante frisar que, independente do tratamento, de modo geral a freqüência das espécies de Eimeria aumentaram após a desmama, exceto para E. canadenses que não se alterou, e para E. wyomingensis cuja freqüência diminuiu de forma acentuada da desmama até o final do estudo (Quadro 1).

Em relação aos resultados obtidos à infecção por Eimeria, nos bezerros, observa-se que na desmama, 30 e $60^{\circ}$ DPD, a média das contagens de OoPG dos animais tratados com lasalocida foi significativamente menor $(\mathrm{P} \leq 0,05)$ que a contagem média de OoPG dos animais do grupo controle. A eficácia da lasalocida contra Eimeria spp. foi de $81,0 \%$, $95,7 \%$ e $97,1 \%$ na desmama, 30 e $60^{\circ}$ DPD, respectivamente (Quadro 2).

Diferença estatística significativa $(\mathrm{P} \leq 0,05)$ em relação ao peso médio dos bezerros controle em comparação aos 
Quadro 1. Percentual de espécies de Eimeria spp. diagnosticadas no pool de amostra de fezes dos bezerros pertencentes aos diferentes grupos experimentais

\begin{tabular}{|c|c|c|c|c|c|c|c|c|}
\hline \multirow{3}{*}{$\begin{array}{l}\text { Espécie de } \\
\text { Eimeria spp. }\end{array}$} & \multicolumn{8}{|c|}{ Dia do estudo/Grupos experimentais } \\
\hline & \multicolumn{2}{|c|}{ Dia zero } & \multicolumn{2}{|c|}{ Desmama } & \multicolumn{2}{|c|}{30 dias pós-desmama } & \multicolumn{2}{|c|}{60 dias pós-desmama } \\
\hline & T01* & $\mathrm{T} 02^{* *}$ & T01* & $\mathrm{T} 02^{* *}$ & T01* & $\mathrm{T} 02^{* *}$ & T01* & T02** \\
\hline E. zuernii & 4,0 & 3,1 & 7,29 & 10,9 & 8,7 & 2,9 & 10 & 4,7 \\
\hline E. ellipsoidalis & 1,0 & 2,1 & ᄀ- & ᄀ- & 1,9 & 1,9 & 8,3 & 1,6 \\
\hline E. cylindrica & ᄀ- & 1,0 & 2,1 & ᄀ- & 1,0 & -า & ᄀ- & -า \\
\hline E. bovis & 6,9 & 6,2 & 9,4 & 31,7 & 22,3 & 11,7 & 28,3 & 12,5 \\
\hline E. canadensis & 5,9 & 7,4 & 6,3 & 12,9 & 8,7 & 16,5 & 15 & 17,2 \\
\hline E. wyomingensis & 72,3 & 32,9 & 10,4 & 25,7 & 10,7 & 4,9 & 15 & 7,8 \\
\hline E. auburnensis & 3,9 & ᄀ- & ᄀ- & ᄀ- & 1,9 & ᄀ- & ᄀ- & ᄀ- \\
\hline E. brasiliensis & 5,9 & 47,4 & 64,5 & 18,8 & 44,7 & 62,1 & 23,4 & 56,2 \\
\hline E. pellita & ᄀ- & 2,1 & 1,1 & 2,0 & ᄀ- & ᄀ- & $-\urcorner$ & ᄀ- \\
\hline
\end{tabular}

* T01 = Bezerros receberam sal mineral proteinado de baixo consumo sem adição de lasalocida.

** T02 = Bezerros receberam sal mineral proteinado de baixo consumo, com adição de lasalocida sódica $1 \mathrm{mg} / \mathrm{kg}$, junto ao creep-feeding.

Quadro 2. Contagens médias (médias aritméticas) de oocistos por grama (OPG) de fezes de Eimeria spp. de bezerros pertencentes aos grupos controle e tratado; resultados da análise de variância e percentuais de eficácia

\begin{tabular}{|c|c|c|c|c|c|c|c|c|c|c|c|}
\hline \multirow[t]{2}{*}{ Dia do estudo } & \multicolumn{8}{|c|}{ Grupos experimentais/Valores médios ${ }^{1}$ das contagens OoPG } & \multirow[b]{2}{*}{$\operatorname{Pr}>F^{2}$} & \multirow{2}{*}{\multicolumn{2}{|c|}{$\frac{\text { Eficácia (\%) }}{\text { T02 }}$}} \\
\hline & \multicolumn{4}{|c|}{ T01: Controle* } & \multicolumn{4}{|c|}{ T02: Tratado** } & & & \\
\hline 0 & 572,83 & 493,48 & 1006,52 & $690,94^{\text {Аa }}$ & 665,56 & 538,01 & 873,14 & $692,25^{\text {Aa }}$ & 0,8552 & 73,77 & - \\
\hline 30 dias pós-desmama & 818,89 & 478,20 & 570,81 & $622,63^{\mathrm{Ab}}$ & 26,04 & 8,77 & 44,96 & $26,60^{\mathrm{Ab}}$ & $<0,0001$ & 86,75 & 95,73 \\
\hline 60 dias pós-desmama & 450,00 & 415,22 & 380,43 & $415,22^{\mathrm{Ac}}$ & 22,34 & 8,70 & 5,16 & $12,06^{\mathrm{Ab}}$ & $<0,0001$ & 117,69 & 97,10 \\
\hline
\end{tabular}

* T01 = Bezerros receberam sal mineral proteinado de baixo consumo sem adição de lasalocida.

** T02 = Bezerros receberam sal mineral proteinado de baixo consumo, com adição de lasalocida sódica $1 \mathrm{mg} / \mathrm{kg}$, junto ao creep-feeding.

${ }^{1}$ Médias seguidas pela mesma letra maiúscula na linha e minuscula na coluna, não diferem entre si $(\mathrm{P}>0,05)$.

${ }^{2}$ Probabilidade de significância do Teste.

${ }^{3}$ Coeficiente de Variação.

Quadro 3. Peso vivo e ganho em peso vivo (kg) de bezerros pertencentes aos grupos controle e tratado; resultados da análise de variância

\begin{tabular}{|c|c|c|c|c|c|c|c|c|c|c|c|c|}
\hline \multirow[t]{2}{*}{ Dias do estudo } & \multirow{2}{*}{$\begin{array}{c}\text { Variável } \\
\text { analisada }\end{array}$} & \multicolumn{8}{|c|}{ Grupos experimentais /Média ${ }^{1}$} & \multirow{2}{*}{$\begin{array}{c}\text { Diferença de } \\
\text { peso (kg) entre } \\
\text { o T02 e T01 }\end{array}$} & \multirow[t]{2}{*}{$\operatorname{Pr}>\mathrm{F}^{2}$} & \multirow[t]{2}{*}{$\mathrm{CV}^{3}$} \\
\hline & & \multicolumn{4}{|c|}{ T01: Controle* } & \multicolumn{4}{|c|}{ T02: Tratado** } & & & \\
\hline Desmama & & 216,3 & 217,0 & 224,3 & $219,2^{\mathrm{A}}$ & 218,4 & 224,2 & 221,3 & $221,3^{A}$ & 2,1 & 0,1453 & 13,52 \\
\hline 30 dias pós-desmama & & 222,5 & 221,7 & 229,1 & $224,4^{\mathrm{B}}$ & 227,1 & 233,1 & 230,1 & $230,0^{A}$ & 5,6 & 0,0002 & 12,92 \\
\hline 60 dias pós-desmama & & 228,2 & 234,1 & 238,5 & $233,6^{\mathrm{B}}$ & 234,6 & 242,2 & 238,9 & $238,6^{A}$ & 5,0 & 0,0003 & 11,47 \\
\hline 60 dias pós-desmama & & 62,8 & 64,6 & 68,3 & $65,2^{\mathrm{B}}$ & 70,5 & 74,4 & 72,2 & $72,4^{\mathrm{A}}$ & 7,2 & 0,0013 & 63,60 \\
\hline
\end{tabular}

* T01 = Bezerros receberam sal mineral proteinado de baixo consumo sem adição de lasalocida.

** T02 = Bezerros receberam sal mineral proteinado de baixo consumo, com adição de lasalocida sódica $1 \mathrm{mg} / \mathrm{kg}$, junto ao creep-feeding.

${ }^{1}$ Médias seguidas pela mesma letra maiúscula na linha, não diferem entre si $(\mathrm{P}>0,05)$.

${ }^{2}$ Probabilidade de significância do Teste.

${ }^{3}$ Coeficiente de Variação.

que receberam lasalocida, via creep-feding na dose de $1 \mathrm{mg} /$ $\mathrm{kg}$, pode ser observada a partir de 30 e 60 DPD. Resultado semelhante foi observado para o ganho em peso vivo médio destes animais, uma vez que, 30 e 60 dias pós-desmama, os bezerros que continuaram sendo tratados com lasalocida ganharam, em média, $7,1 \mathrm{~kg}$ e $7,2 \mathrm{~kg}$ a mais que os bezerros do grupo controle, respectivamente (Quadro 3).

Como no início do estudo havia animais de quatro a seis meses de idade, e estes permaneceram no estudo até completarem 10 meses, é possível analisar a eficácia do ativo utilizado, e o ganho em peso médio dos bezerros levando em consideração o período em que os animais ingeriram a lasalocida. Em outras palavras, os animais com 6 meses de idade no início do estudo ingeriram lasalocida durante quatro meses, enquanto que os bezerros com cinco meses, ingeriram este mesmo produto por cinco meses, e por sua vez os bezerros com quatro meses de idade ingeriram a lasalocida por seis meses. Sendo assim, verifica-se que o referido composto demonstrou eficácia $\geq 95 \%$, naqueles bezerros que ingeriram a lasalocida por pelo menos cinco 
Quadro 4. Contagens médias (médias aritméticas) de oocistos por grama de fezes (OPG) de Eimeria spp. de bezerros pertencentes aos grupos controle e tratado, levando em consideração o período em meses de ingestão da lasalocida pelos animais tratados; resultados da análise de variância e percentuais de eficácia

\begin{tabular}{|c|c|c|c|c|c|}
\hline \multirow{2}{*}{$\begin{array}{l}\text { Tempo (meses) } \\
\text { de ingestão da } \\
\text { lasalocida pelos } \\
\text { animais tratados }\end{array}$} & \multicolumn{2}{|c|}{$\begin{array}{c}\text { Grupos experimentais / Valores médios }{ }^{1} \\
\text { das contagens de oocistos de OoPG }\end{array}$} & \multirow[t]{2}{*}{$\operatorname{Pr}>F^{2}$} & \multirow[t]{2}{*}{$\mathrm{CV}^{3}$} & \multirow[t]{2}{*}{$\begin{array}{l}\text { Eficácia }(\%) \\
\text { T02 }\end{array}$} \\
\hline & T01: Controle* & T02: Tratado** & & & \\
\hline 2 & $616,67^{A}$ & $71,59^{\mathrm{B}}$ & $<0,0001$ & 65,45 & 88,4 \\
\hline 3 & $514,90^{\mathrm{A}}$ & $59,22^{\mathrm{B}}$ & $<0,0001$ & 67,80 & 88,5 \\
\hline 4 & $538,52^{\mathrm{A}}$ & $74,69^{\mathrm{B}}$ & $<0,0001$ & 69,76 & 86,1 \\
\hline 5 & $476,06^{\mathrm{A}}$ & $15,28^{\mathrm{B}}$ & $<0,0001$ & 69,80 & 96,8 \\
\hline 6 & $488,33^{\mathrm{A}}$ & $12,50^{\mathrm{B}}$ & $<0,0001$ & 67,70 & 97,4 \\
\hline
\end{tabular}

* T01 = Bezerros receberam sal mineral proteinado de baixo consumo sem adição de lasalocida.

** T02 = Bezerros receberam sal mineral proteinado de baixo consumo, com adição de lasalocida sódica $1 \mathrm{mg} / \mathrm{kg}$, junto ao creep-feeding.

${ }^{1}$ Médias seguidas pela mesma letra maiúscula na linha, não diferem entre si $(\mathrm{P}>0,05)$.

${ }^{2}$ Probabilidade de significância do Teste.

${ }^{3}$ Coeficiente de Variação.

Quadro 5. Peso vivo e ganho em peso vivo (kg) de bezerros pertencentes aos grupos controle e tratado, levando em consideração o período em meses de ingestão da lasalocida pelos animais tratados; resultados da análise de variância

\begin{tabular}{|c|c|c|c|c|c|c|}
\hline \multirow{2}{*}{$\begin{array}{l}\text { Tempo (meses) } \\
\text { de ingestão da } \\
\text { lasalocida pelos } \\
\text { animais tratados }\end{array}$} & \multirow{2}{*}{$\begin{array}{l}\text { Variável } \\
\text { analisada }\end{array}$} & \multicolumn{2}{|c|}{ Grupos experimentais/Média ${ }^{1}$} & \multirow{2}{*}{$\begin{array}{c}\text { Diferença de } \\
\text { peso (kg) entre }\end{array}$} & \multirow[t]{2}{*}{$\operatorname{Pr}>F^{2}$} & \multirow[t]{2}{*}{$\mathrm{CV}^{3}$} \\
\hline & & T01: Controle* & $\begin{array}{c}\text { T02: Tratado** } \\
\text { o T02 e T01 }\end{array}$ & & & \\
\hline 2 & Peso vivo (kg) & $239,9^{A}$ & $241,3^{A}$ & 1,4 & 0,9885 & 11,66 \\
\hline 3 & & $217,7^{\mathrm{A}}$ & $214,9^{\mathrm{A}}$ & $-2,8$ & 0,4808 & 13,46 \\
\hline 4 & & $218,3^{A}$ & $225,7^{\mathrm{A}}$ & 7,4 & 0,2544 & 14,23 \\
\hline 5 & & $220,5^{\mathrm{B}}$ & $228,1^{\mathrm{A}}$ & 7,6 & 0,0006 & 11,32 \\
\hline 6 & & $222,6^{\mathrm{B}}$ & $237,0^{A}$ & 14,4 & 0,0093 & 11,32 \\
\hline 2 & Ganho em & $41,4^{\mathrm{A}}$ & $45,9^{\mathrm{A}}$ & 4,5 & 0,7654 & 11,66 \\
\hline 3 & peso vivo (kg) & $52,1^{\mathrm{A}}$ & $54,7^{\mathrm{A}}$ & 2,6 & 0,4568 & 13,46 \\
\hline 4 & & $52,0^{\mathrm{A}}$ & $60,0^{\mathrm{A}}$ & 8,0 & 0,26789 & 14,23 \\
\hline 5 & & $69,5^{\text {в }}$ & $78,4^{A}$ & 8,90 & 0,0036 & 11,32 \\
\hline 6 & & $83,5^{\text {в }}$ & $97,0^{\mathrm{A}}$ & 13,5 & 0,0102 & 11,32 \\
\hline
\end{tabular}

Ganho em peso vivo (kg) de bezerros pertencentes aos grupos controle e tratado, levando em consideração apenas os 15 animais do grupo controle que não apresentaram oocistos de Eimeria e ovos de helmintos (estrongilídeos) durante todo o experimento.

\begin{tabular}{|c|c|c|c|c|c|}
\hline Desmama & Ganho em & 53,5 & 54,9 & 1,4 & Não se aplica \\
\hline 30 dias pós-desmama & Peso vivo (kg) & 62,8 & 63,0 & 0,3 & \\
\hline 60 dias pós-desmama & & 74,2 & 72,4 & $-1,8$ & \\
\hline
\end{tabular}

* T01 = Bezerros receberam sal mineral proteinado de baixo consumo sem adição de lasalocida.

** T02 = Bezerros receberam sal mineral proteinado de baixo consumo, com adição de lasalocida sódica $1 \mathrm{mg} / \mathrm{kg}$, junto ao creep-feeding.

${ }^{1}$ Médias seguidas pela mesma letra maiúscula na linha, não diferem entre si $(\mathrm{P}>0,05)$.

${ }^{2}$ Probabilidade de significância do Teste.

${ }^{3}$ Coeficiente de Variação.

meses, apesar de que depois de dois meses de ingestão deste ativo pelos bezerros, este composto demonstrou eficácia de 88,4\% contra Eimeria. 0 ganho em peso dos animais pertencentes ao grupo que recebeu lasalocida foi significativamente $(\mathrm{P} \leq 0,05)$ mais elevado, em comparação ao grupo controle, naqueles bezerros que ingeriram o referido composto por pelo menos cinco meses (Quadro 4 e 5).

No que diz respeito às contagens de OPG (estrongilídeos) dos bezerros, após o início do tratamento de todos os bezerros a cada 45 dias (moxidectina $200 \mu \mathrm{g} / \mathrm{kg}$ ) a partir do dia zero do estudo, os animais demonstraram um baixo parasitismo por estrongilídeos, quando as contagens médias de OPG após o início do estudo foi $\leq 4,8$ OPG. Antes do início do experimento, as contagens médias de OPG (estrongilídeos) quantificadas nos animais do T01 e T02 foi de 321,1 e 434,9 OPG, respectivamente.

Em função dos animais terem sido alocados de forma aleatória aos grupos de tratamentos/repetição de área, 15 bezerros pertencentes ao Tratamento 01 (grupo controle, sendo 7 fêmeas e 8 machos) não apresentaram oocistos de Eimeria nas fezes em todas as datas do estudo. Quando analisamos os dados referentes as pesagens destes 15 animais (isentos de infecção por Eimeria e que não receberam lasalocida), verifica-se que estes bezerros apresentaram ganho em peso médio de $62,8 \mathrm{~kg}$ e $74,2 \mathrm{~kg}$ no $30^{\circ}$ e $60^{\circ}$ DPD, respectivamente. Já os bezerros pertencentes ao T02 (tratados com lasalocida via creep-feeding), demonstraram, 
nas mesmas datas supracitadas, um ganho em peso médio de $63,0 \mathrm{~kg}$ e $72,4 \mathrm{~kg}$, respectivamente (Quadro 5).

Pelo Quadro 6, é possível verificar que não houve diferença estatística significativa $(P>0,05)$ nas contagens médias de OoPG dos bezerros machos, em comparação as contagens médias de oocistos do protozoário em questão quantificados nas bezerras fêmeas, durante todo estudo. Por outro lado, foi possível observar que as contagens médias de OoPG de ambos os sexos, diminuíram de forma significativa $(\mathrm{P} \leq 0,05) 60$ dias pós-desmama.

\begin{tabular}{|c|c|c|c|c|}
\hline Dia do estudo & $\begin{array}{l}\text { Valores médio } \\
\text { oocistos de Eim } \\
\text { de fezes (OPG) }\end{array}$ & $\begin{array}{l}\text { s contagens de } \\
\text { spp. por grama } \\
\text { relação ao sexo }\end{array}$ & $\operatorname{Pr}>F^{2}$ & \\
\hline & Machos & Fêmeas & & \\
\hline 0 & 701,1Aa & $679,2 \mathrm{Aa}$ & $<0,08901$ & 45,67 \\
\hline Desmama & $597,7 \mathrm{Aa}$ & $530,7 \mathrm{Aa}$ & $<0,7453$ & 43,32 \\
\hline 30 dias pós-desmama & $607,7 \mathrm{Aa}$ & 637,7Аa & $<0,8761$ & 46,78 \\
\hline 60 dias pós-desmama & $380,7 \mathrm{Ab}$ & $449,7 \mathrm{Ab}$ & $<0,5437$ & 49,80 \\
\hline $\begin{array}{l}\text { Probabilidade de signi- } \\
\text { ficância do teste }\end{array}$ & $<0,0001$ & $<0,0001$ & & \\
\hline Coeficiente de variação & 68,78 & 69,87 & & \\
\hline
\end{tabular}

${ }^{1}$ Médias seguidas pela mesma letra maiúscula na linha e minúscula na coluna, não diferem entre si $(\mathrm{P}>0,05)$.

${ }^{2}$ Probabilidade de significância do Teste.

${ }^{3}$ Coeficiente de Variação.

\section{DISCUSSÃO}

A diferença no ganho em peso entre os bovinos que receberam lasalocida em comparação aos animais pertencentes aos grupos controle (sal proteinado sem a adição de lasalocida), pode ser justificada, em partes, pelo fato do ativo em questão apresentar ação sobre a população microbiana do rúmen, o que por sua vez melhora a eficiência alimentar, e favorece o ganho em peso dos animais (Thonney et al. 1981). Estudos foram realizados em bezerros leiteiros, demonstrando o benefício do seu uso contra oocistos de Eimeria, bem como em um ligeiro incremento no desenvolvimento ponderal destes animais (Waggoner et al. 1994, Quigley et al. 1997, Goodier et al. 2012). Outros estudos com a lasalocida demonstraram, ainda, que a utilização deste ativo melhorou o desenvolvimento ponderal de novilhos de corte em terminação (Thonney et al. 1981, Restle et al. 1999), ou ainda a produção de leite das vacas (Bos taurus) que receberam este ativo (Higginbotham et al. 2010). Em propriedades que tem como objetivo a venda de bezerros logo após a desmama, recomenda-se o início do tratamento com a lasalocida, junto ao creep-feeding, a partir de três messes de idade, uma vez que diferencial no ganho em peso médio dos bezerros tratados foi significativamente $(\mathrm{P} \leq 0,05)$ mais elevado, em comparação ao grupo controle, após cinco meses de tratamento com o referido composto.

Apesar do exposto, deve-se ressaltar que no presente estudo, em função dos animais terem sido alocados aos grupos de tratamentos de forma aleatória, 15 bezerros pertencentes ao grupo não tratado (controle - T01) não apre- sentaram infecção por Eimeria durante todo o experimento. Além disso, eles receberam moxidectina $200 \mu \mathrm{g} / \mathrm{kg}$ no dia 0 e a cada 45 dias após o início do estudo, o que também proporcionou contagens negativas de OPG (estrongilídeos) destes animais durante o estudo. Desta forma, observando o ganho em peso médio destes 15 bezerros, em comparação ao grupo que recebeu lasalocida, verifica-se que estes animais isentos de Eimeria e que não receberam lasalocida, apresentaram desenvolvimento ponderal muito semelhante aos animais tratados com lasalocida. Apesar de não ter sido possível realizar uma análise estatística destes dados, em função da diferença do número de animais avaliados em cada grupo, é possível relacionar a diferença no ganho de peso entre os grupos controle e tratado, não apenas ao uso da lasalocida, mas também, em partes, a infecção dos animais por Eimeria. Entretanto, futuros estudos devem ser realizados para comprovar esta hipótese.

Sete das 9 espécies de Eimeria relatadas no presente estudo foram também detectadas em bovinos de São Paulo, Bahia e Minas Gerais (Rebouças et al. 1994, Almeida et al. 2011, Bruhn et al. 2011, 2012). A predominância das espécies identificadas neste estudo é diferente das encontradas na literatura (Rebouças et al. 1994, Almeida et al. 2011, Bruhn et al. 2011, 2012). Os referidos pesquisadores relatam que E. bovis e E. zuernii foram as mais prevalentes e consequentemente importantes em bovinos. No presente estudo, E. brasiliensis, seguida de E. wyomingensis e E. bovis foram as espécies que mais predominaram. Apesar de ser notório que Eimeria esta disseminada por todo Brasil (Lima 2004), inexistem trabalhos realizados no estado de Goiás, o que dificulta a discussão dos resultados encontrados. De qualquer maneira, futuros estudos devem ser realizados para entender o porque desta diferença nestes estados brasileiros.

Outro aspecto interessante, é que a maior incidência desta enfermidade tem sido reportada em animais jovens, confinados ou em bezerros criados em sistemas altamente tecnificados que adotam a inseminação artificial em tempo fixo no manejo reprodutivo, com o intuito de se aumentar a produtividade (Lima 2004, Bruhn et al. 2011, 2012). No entanto, no presente estudo, os bezerros pertencentes ao grupo controle (que apresentavam contagens de OoPG elevadas) eram criados à pasto, e a reprodução utilizada na propriedade era realizada por meio de monta natural, sem uma estação de monta definida. Por outro lado, alguns pesquisadores relatam a elevada prevalência de Eimeria em animais adultos criados de forma extensiva (Cavalcante et al. 2009), entretanto, estes autores enfatizam que tal fato pode acontecer, principalmente se estes animais forem submetidos a uma alimentação pobre em proteínas, ou se forem submetidos a situações de estresse, ou ainda quando existem problemas de manejo na propriedade que acabam interferindo no estado imunológico dos animais. É importante ressaltar que no presente trabalho, os animais receberam sal proteinado, e a taxa de lotação animal durante o estudo foi de aproximadamente 1,5 UA/hectare. Um fator que pode ter influenciado no grau de infecção dos animais por Eimeria, é a época do ano em que o estudo foi realizado. 
É notório que o desmame predispõe os animais a uma situação de estresse, o que por sua vez poderá desencadear no aumento do grau de infecção por parasitos de um modo geral (Lima 2004, Mundt et al. 2003, Lopes et al. 2013, Philippe et al. 2014, Heckler et al. 2016). Entretanto, no presente estudo foi observada diminuição nas contagens de OoPG após o desmame dos animais. Ao observamos os resultados das contagens de OoPG no presente estudo, verifica-se que as contagens médias de OoPG após a desmama já eram mais baixas em comparação a primeira data de avaliação, e estes valores diminuíram ainda mais aos 60 DPD.

\section{CONCLUSÕES}

Nas condições do presente experimento a lasolocida foi eficaz ( $\geq 95 \%$ ) contra a infecção por Eimeria em bezerros Nelore e ocasionou um aumento do ganho de peso nos animais tratados $(7,2 \mathrm{~kg}$ a mais - $\mathrm{p} \leq 0,05)$, em comparação ao controle.

Apesar de a lasalocida ser utilizada como um aditivo alimentar para animais, a diferença no ganho em peso vivo médio entre animais tratados com a lasalocida, em comparação a animais pertencentes ao grupo controle, também pode ser relacionada, em partes, a infecção dos animais por Eimeria spp., conforme discutido neste artigo, entretanto, futuros estudos devem ser conduzidos para comprovar esta hipótese.

Foram identificadas nove espécies de Eimeria nos bezerros em ordem decrescente: E. brasiliensis, E. wyomingensis, E. bovis, E. canadenses, E. zuernii, E. auburnensis, E. ellipsoidalis, E. pellita e E. cylindrica.

Tanto o sexo dos bezerros, quanto o processo de desmame não influenciaram na carga parasitária destes animais por Eimeria $(\mathrm{P}>0,05)$.

\section{REFERÊNCIAS}

Almeida V.A., Magalhães V.C., Neta E.S.M. \& Munho A.D. 2011. Frequency of species of the genus Eimeria in naturally infected cattle in Southern Bahia, Northeast Brazil. Revta Bras. Parasitol. Vet. 20:78-81.

Aumont G., Yvore P. \& Esnault A. 1984. Coccidiosis in goats: experimental model effects of parasitism on the feeding behaviour and the growth of animals and intestinal lesions. Annal. Res. Vet. 15:467-473.

Bruhn F.R., Lopes M.A., Demeu F.A., Perazza C.A., Pedrosa M.F. \& Guimarães A.M. 2011. Frequency of species of Eimeria in females of the holstein-friesian breed at the post-weaning stage during autumn and winter. Revta Bras. Pasitol. Vet. 20:303-307.

Bruhn F.R.P., Silva Junior F.A., Carvalho A.H.O., Orlando D.R., Rocha C.M.B.M \& Guimarães A.M. 2012. Occurrences of Eimeria spp. and gastrointestinal nematodes in dairy calves in southern Minas Gerais, Brazil. Revta Bras. Parasitol. Vet. 21:175-177.

Cavalcante A.C.R., Vieira L.S., Chagas A.C.S. \& Molento M.B. 2009. Doenças parasitárias de caprinos e ovinos. Epidemiologia e controle. Informação Tecnológica, Embrapa, Brasília. 603p.

Daugschies A. \& Najdrowski M. 2005. Eimeriosis in cattle: current understanding. J. Vet. Med. 52:417-427.

Duszynski D. \& Wilber P.G. 1997. A guideline for the preparation of species descriptions in the Eimeriidae. J. Parasitol. 83:333-336.

Goodier G.E., Williams J.C., O’Reilly K.L., Snider T.G., Stanley C.C., Dolejsiova A.H. \& Williams C.C. 2012. Effects of supplemental vitamin E and lasao- cid on growth and immune responses of calves challenged with Eimeria bovis. Profess. Anim. Scient. 28:97-107.

Gordon H.M. \& Whitlock H.V. 1939. A new technique for counting nematode eggs in sheep faeces. Commonw. Scient. Indust. Org. 12:50-52.

Higginbotham G.E., Pereira L.N., Chebel R.C. \& Lehenbauer T.W. 2010. A field trial comparing the effects of supplementation with aureomycin plus lasalocid or monensin on the health and production performance of dairy calves. Profess. Anim. Sci. 26:520-526.

Heckler R.P., Borges G.G.L., Vieira M.C., Conde M.H., Green M., Amorin M.L., Echevarria J.T., Oliveira T.L., Moro E., Onselen V.J. \& Borges F.A. 2016. New approach for the strategic control of gastrointestinal nematodes in grazed beef cattle during the growing phase in central Brazil. Vet. Parasitol. 221:123-129.

Lima J.D. 2004. Coccidiose dos ruminantes domésticos. Revta Bras. Parasitol. Vet. 13: 9-13.

Lima V.X.M., Ahid S.M.M. \& Simplicio A.A. 2009. Efeito de sal mineral enriquecido ou não com ionóforos sobre a frequência de eimeriídeos de fêmeas caprinas jovens. Revta Agroneg. Meio. Amb. 2:63-71.

Lopes W.D.Z., Santos T.R., Sakamoto C.A., Valarelli R.L., Paiva P. \& Costa A.J. 2013. Persistent efficacy of $3.5 \%$ doramectin compared to $3.15 \%$ ivermectin against gastrointestinal nematodes in experimentally-infected cattle in Brazil. Res. Vet. Sci. 94:290-294.

Lopes W.D.Z., Carvalho R.S., Pereira V., Martinez A.C., Cruz B.C., Teixeira W.F.P., Maciel W.G., Costa A.J., Soares V.E., Lino D.G.A., Rodriguez F.S. \& Borges F.A. 2014. Efficacy of sulfadoxine + trimethoprim compared to management measures for the control of Eimeria parasitism in naturally infected and clinically asymptomatic sheep that were maintained in a feedlot. Small Rum. Res. 116:37-43.

Mundt H.C., Daugschies A., Vebe F. \& Rinke M. 2003. Efficacy of toltrazuril against artificial infection with Eimeria bovis in calves. Parasit. Res. 9:s166-s167.

Nogueira V.A., França T.N. \& Peixoto P.V. 2009. Intoxicação por antibióticos ionóforos em animais. Pesq. Vet. Bras. 29:191-197.

Nogueira A.P.A., Souza R.I.C., Santos B.S., Pinto A.P., Ribas N.L.K.S., Lemos R.A.A. \& Santana F.J.F. 2010. Polioencefalomalacia experimental induzida por amprólio em bovinos. Pesq. Vet. Bras. 30:631-636.

Philippe P., Alzieu J.P., Taylor M.A. \& Drochies P.H. 2014. Comparative efficacy of diclazuril (Vecoxan ${ }^{\circledR}$ ) and toltrazuril (Baycox bovis ${ }^{\circledR}$ ) against natural infections of Eimeria bovis and Eimeria zuernii in French calves. Vet. Parasitol. 206:129-137.

Quigley J.D., Drewrym J.J., Murray L.M. \& Ivey S.J. 1997. Effects of lasalocid in milk replacer or calf starter on health and performance of calves challenged with Eimeria species. J. Dairy Sci. 80:2972-2976.

Rebouças M.M., Grasso Sposito-Filha S., Amaral V., Santos S.M. \& Lima D. 1994. Prevalência e distribuição de protozoários do gênero Eimeria (Apicomplexa: Eimeriidae) em bovinos nos municípios de Altinópolis, Taquaritinga, São Carlos e Guairá - Estado de São Paulo, Brasil. Revta Bras. Parasit. Vet. 3:125-130.

Restle J., Soares A.B., Back M.V., Brondani I.V. \& Calça K.G. 1999. Suplementação associada com lasalocida para novilhos em terminação em pastagem cultivada de inverno. Ciência Rural 29:555-559.

Rutkowski J. \& Brzezinski B. 2013. Structures and properties of naturally occurring polyether antibiotics. BioMed. Res. Int. 162513:31-32.

SAS Institute 1989-1996. SAS ${ }^{\circledR}$ User's Guide: estatistics. SAS Institute Inc., Cary, NC, USA.

Thonney E., Heide K. \& Duhaime D.J. 1981. Growth, feed efficiency and metabolite concentration of cattle feed high fo-rage diets with lasalocid or monensin supplements. J. Anim. Sci. 52:427-433.

Ueno H. \& Gonçalves P.C. 1998. Manual para diagnóstico das helmintoses de ruminantes. J. IICA 10:1-65.

Waggoner J.K., Cecava M.J. \& Kazacos K.R. 1994. Efficacy of lasalocid and decoquinate against coccidiosis in naturally infected dairy calves. J. Dairy Sci. 77:349-353. 